\title{
El uso de armas nucleares bajo examen: ¿Es legal su uso en situaciones de conflicto armado? ${ }^{(*)(*)}$
}

\author{
The use of nuclear weapons under review: Is their use legal in situations \\ of armed conflict?
}

\author{
Alessandra Enrico Headrington ${ }^{(* *)}$ \\ Pontificia Universidad Católica del Perú (Lima, Perú)
}

Resumen: El cuestionamiento sobre el ensayo y/o uso de armas nucleares en conflictos armados y las consecuencias ya advertidas en los ataques a Hiroshima y Nagasaki han puesto de manifiesto no solo las trágicas secuelas personales, medioambientales y materiales que estas tuvieron principalmente en quienes no toman parte en las hostilidades. Por ello, la discusión sobre su uso como medio de conflicto armado, resulta una discusión vigente que puede evitar trágicos desenlaces como los mostrados durante la segunda guerra mundial. De ahí la importancia de analizar que su potencial uso devendría en ilícito por contravenir un conjunto de normas en el Derecho Internacional, pero particularmente en el Derecho Internacional Humanitario.

En esa medida, es posible discutir su utilización en contextos en donde se evalúa la legalidad del uso de la fuerza (ius ad bellum), como en situaciones de conflictos armados (ius in bello). En el presente artículo se abordará exclusivamente este último punto, y se analizarán las consecuencias jurídicas a la luz del Derecho Internacional Humanitario y tratados específicos sobre la materia. De igual manera, esta investigación plantea la realización de un examen más específico sobre tres principios clave durante la conducción de hostilidades: la distinción, proporcionalidad y necesidad militar, que permiten dilucidar -de manera técnica y objetiva- si su utilización puede o no ser lícita para atacar a la parte adversaria.

La conclusión, se adelanta, es negativa, el ensayo y/o uso de armas nucleares y la tendencia hacia la no proliferación, el desarme y prohibición son mandatos -a la fechainjustificables desde una perspectiva jurídica. Y su contravención no solo deviene en una violación grave al Derecho Internacional Humanitario, sino que eventualmente podría conllevar a la determinación de responsabilidad penal individual para quienes ordenen y/o ejecuten ataques con armas prohibidas cuyos efectos sean indiscriminados como el que genera un arma nuclear.

Palabras clave: Derecho Internacional Humanitario - Normas consuetudinarias del DIH - Convenios de Ginebra - Principio de humanidad - Armas nucleares - Tratados No proliferación - Hiroshima - Nagasaki - Necesidad militar

(*) Nota del Equipo Editorial: Este artículo fue recibido el 28 de octubre de 2021 y su publicación fue aprobada el 1 de diciembre de 2021.

${ }^{* *}$ La autora desea agradecer a Jimena Soto Rojas, miembro del Grupo Interdisciplinario de Investigación en Derechos Humanos (GRIDEH), por su invaluable apoyo como asistenta de investigación para la elaboración de este artículo.

$\left(^{(* *)}\right.$ Abogada por la Pontificia Universidad Católica del Perú (PUCP) con estudios en la Escuela de Derecho de la Universidad Carlos III de Madrid. Es Magíster en Derechos Humanos de la PUCP. Ha trabajado en la Comisión Especial para los Refugiados del Perú y más recientemente como asociada legal de ACNUR Perú. Actualmente es docente en la Facultad de Derecho de la PUCP, especialista en Derecho Internacional de los Derechos Humanos, Derecho Internacional Humanitario y Derecho Internacional de los Refugiados. ORCID: https://orcid.org/0000-0002-4260-5679. Correo electrónico: alessandra.enrico@pucp.edu.pe. 


\begin{abstract}
The discussion regarding nuclear weapons uses or tests in the context of an armed conflict, and the subsequent consequences that have already been noticed in the attacks on Hiroshima and Nagasaki, poses several humanitarian challenges in relation to the tragic personal, environmental, and material costs for those who do not take part in the hostilities: the civilian population. Therefore, it become important to conduct an assessment to develop its illegality under international law but specifically under International Humanitarian Law.
\end{abstract}

To that extent, it is possible to analyze its use in contexts where the legality of the use of force is examined (ius ad bellum), as well as in situations of armed conflict (ius in bello). This latter view is fully discussed in the present article. Thus, the legal consequences in the light of International Humanitarian Law and specific treaties on the matter are thoroughly developed. This includes the step-bystep assessment of three key principles: distinction, proportionality, and military necessity, which allow to objectively elucidate whether the use of nuclear weapons may or may not be lawful during the conduction of hostilities.

In summary, the answer to its legality is negative. The use or tests of nuclear weapons and the trend towards the nonproliferation, disarmament, and more lately its complete prohibition is unquestionable from a legal approach. Additionally, its contravention not only becomes a serious violation of International Humanitarian Law but could eventually lead to the determination of individual criminal responsibility, for those who order and/or execute attacks with prohibited weapons whose effects are indiscriminate, as nuclear weapons are.

Keywords: International Humanitarian Law - IHL customary rules - Geneva Conventions - Humanitarian principle - Nuclear weapons - Treaties - Non-proliferation - Hiroshima - Nagasaki Military necessity

\section{Introducción}

Cuando se cuestiona el uso de armas nucleares en situaciones en donde se aplica el Derecho Internacional Humanitario (en adelante "DIH"), se hace referencia a la prohibición de determinados medios en la conducción de hostilidades que no se dirigen exclusivamente a generar una ventaja militar para alguna de las partes, sino que cuyos efectos resultarían indiscriminados. Esto quiere decir que causarían un daño mucho mayor en relación a la ventaja militar que se busca obtener.

A modo de ejemplo, las explosiones nucleares de Hiroshima y Nagasaki, en el contexto de la segunda guerra mundial pusieron en relieve el efecto generalizado y devastador que supone el uso de un arma nuclear. Sin embargo, pese al impacto y a los testimonios de los sobrevivientes de dicho ataque (los
Hibakusha), la producción de armas nucleares y su uso en ensayos en la actualidad, denotan la potencial posibilidad de emplearlas en situaciones de conflicto armado. De ahí la relevancia de discutir este tema desde una perspectiva jurídica que permita evidenciar la necesidad de dotar de mayor contenido a los criterios elementales de humanidad que protegen la dignidad del individuo y que se encuentran cristalizados en el DIH.

En ese sentido, para poder llevar a cabo un análisis objetivo sobre la legalidad del uso de armas nucleares es imprescindible comprender, en primer lugar, el marco jurídico del DIH que aborda la limitación y/o prohibición general de medios o armas durante las hostilidades. En este punto, se evaluará el cuerpo jurídico que los regula, lo que incluye los Convenios de Ginebra, sus Protocolos adicionales y las normas consuetudinarias del DIH. Se debe adelantar, no obstante que, si bien no se advierte una prohibición específica, todo este cuerpo jurídico expone mandatos claros que permiten una interpretación que no admitiría su utilización.

En un segundo punto, se revisarán los alcances de la definición de un arma nuclear y los usos comunes que se les da, en particular para temas pacíficos y militares. En relación a este último, se desarrollará el impacto letal de estas armas durante conflictos armados y la importancia de hacer un análisis ex ante sobre los efectos, incluso a largo plazo, que estas pueden tener. Se estudiará el caso del conflicto armado no internacional entre Estados Unidos y Japón para ilustrar las consecuencias inmediatas y a largo plazo de su uso.

En un tercer punto se estudiará el largo camino de desarrollo convencional hacia la regulación de armas nucleares hasta llegar a su último y más vigente estadio: la prohibición total. En esa línea, se revisarán los principales tratados sobre la materia y la cristalización de normas relativas a la no proliferación y desarme cuya contribución es fruto de las negociaciones interestatales. Se destacará como principal óbice para la implementación la falta de ratificación universal de estos instrumentos jurídicos, y algunos episodios que evidencian un compromiso real por parte de los Estados con arsenal nuclear. 
Como cuarto y último aspecto se abordará el posicionamiento de instituciones jurídicas y organismos humanitarios sobre la necesidad de prohibir el uso de armas nucleares. Por ejemplo, se revisará un pronunciamiento realizado por la Corte Internacional de Justicia (en adelante "ClJ o la Corte") en el marco de su competencia consultiva que, sin determinar finalmente la prohibición absoluta del uso de armas nucleares, concluyó cuáles deben de ser las consideraciones mínimas por seguir antes del uso de determinadas armas. En la misma línea, se verá el documento de posicionamiento del Comité Internacional de la Cruz Roja (en adelante "CICR") sobre los retos contemporáneos del DIH, en particular en lo tocante al uso de armas nucleares. Y con ello presente, se examinará paso a paso la ilegalidad del uso de estas armas, y si su utilización contraviene o no principios elementales del DIH como distinción, proporcionalidad y necesidad militar.

\section{La elección de medios en el DIH: La evolución de parámetros normativos para su limitación y prohibición}

A diferencia del ius ad bellum en donde se discute la legalidad de la amenaza o el uso de la fuerza contra un Estado, conforme a la Carta de Naciones Unidas (art. 2.4), en el ius in bello el principal objetivo es dotar de contenidos mínimos de humanidad a situaciones de conflictos armados, las cuales muchas veces se encuentran sobrepasadas por la acción militar. Para ello, una serie de reglas y disposiciones se han establecido con miras a limitar el impacto del uso de armas y racionalizar una situación que, por su naturaleza, impone objetivos militares y permite el despliegue de ataques armados. De este modo, en el DIH el fin último es que, en medio del fuego cruzado de los ataques, las partes en conflicto tengan presente que el núcleo duro e intransigible es el respeto y preservación de la dignidad del ser humano, participe o no de los enfrentamientos.

Para cumplir con tales fines, la conducción de hostilidades entre las partes tiene parámetros claros de limitación y/o prohibición de aquellos medios (armas) y métodos (estrategias) de guerra que por sus efectos causen daños superfluos o sufrimientos innecesarios. Precisamente, una de las primeras manifestaciones jurídicas que limitan la acción de las partes en un conflicto armado es la cláusula Martens. Su importancia radica en que se trata de una de las primeras salvaguardas que contiene términos claros para las partes enfrentadas. Esta fue incorporada por primera vez en el Preámbulo del II Convenio de La Haya relativo a las leyes y costumbres de la guerra terrestre y establecía que:

En los casos no comprendidos en las disposiciones reglamentarias adoptadas por ellas, las poblaciones y los beligerantes permanecen bajo la garantía y el régimen de los principios del
Derecho de Gentes preconizados por los usos establecidos entre las naciones civilizadas, por las leyes de la humanidad y por las exigencias de la conciencia pública (1899).

Los alcances de esta disposición se erigen como un remedio jurídico a ciertas acciones que surgen de un contexto de guerra y no están reguladas por normas convencionales (Salmón, 2012). De este modo, permite que se rijan bajo Derecho Consuetudinario y por las leyes de humanidad. En el plano práctico, es decir en el teatro de operaciones en donde se despliegan los ataques armados, esta Cláusula sirve como un freno a la acción de las partes, limitando sus decisiones y el impacto de sus acciones partir del principio de humanidad que constituye la piedra angular en el DIH.

Con el paso de los años, este desarrollo primigenio ofrecido por la cláusula Martens fue extendiéndose hasta la identificación de algunos hitos importantes que dieron un espaldarazo al principio de humanidad. El primero es el pronunciamiento de la $\mathrm{ClJ}$ respecto del caso "Canal de Corfú", en donde reafirmó la relevancia del principio, señalando que "aquellas consideraciones elementales de humanidad son aún más fundamentales en tiempo de paz que en tiempos de guerra" (1949). El segundo hito se dio ese mismo año con la aprobación de los Convenios de Ginebra (1949), y posteriormente sus Protocolos Adicionales en 1977 , todas las cuales fueron precisando de manera más puntual los alcances sobre el empleo de armas en el campo de batalla, e incluso qué armas deberían de estar prohibidas debido a sus efectos indiscriminados. Con el paso de los años, la aprobación de un conjunto de normas que recogían el carácter consuetudinario del DIH fueron recogiendo estos preceptos aplicables, en su gran mayoría, a ambos tipos de conflicto.

A modo de síntesis, el siguiente cuadro contempla las disposiciones específicas contenidas en los Convenios de Ginebra de 1949, sus Protocolos Adicionales de 1977, y la normativa consuetudinaria del DIH que limita el uso de determinadas armas de acuerdo al tipo de conflicto armado: 


\begin{tabular}{|c|c|c|}
\hline & Normas convencionales & Normas consuetudinarias \\
\hline $\begin{array}{l}\text { Conflictos armados } \\
\text { internacionales }\end{array}$ & 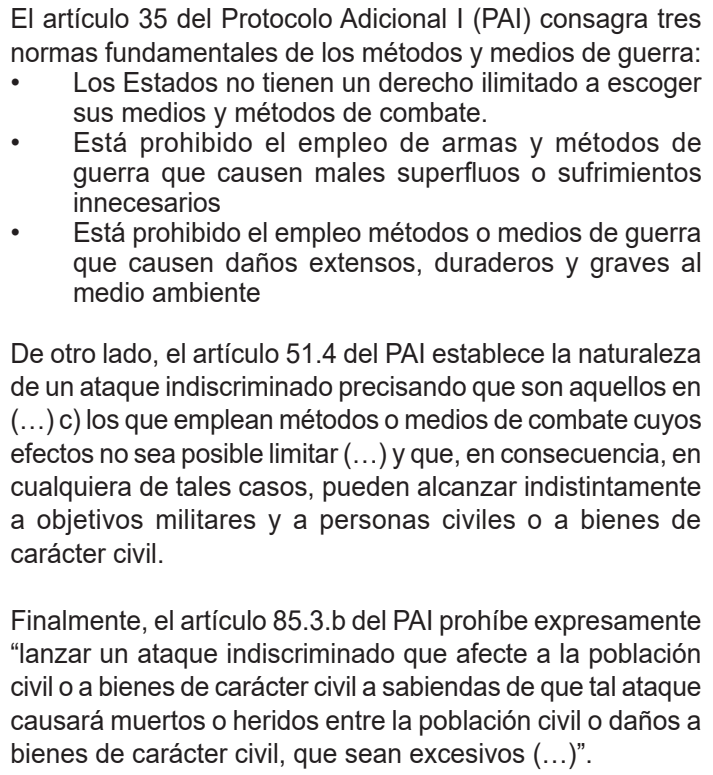 & $\begin{array}{l}\text { En ambos tipos de conflicto armado el DIH } \\
\text { consuetudinario prohíbe el uso de ataques } \\
\text { indiscriminados, y de determinadas armas. } \\
\text { Por ejemplo, la norma 11, } 12 \text { y } 13 \text { del DIH } \\
\text { consuetudinario establece la prohibición de } \\
\text { ataques indiscriminados. Es decir, (a) que no } \\
\text { están dirigidos contra un objetivo militar concreto; } \\
\text { (b) en los que se emplean métodos o medios } \\
\text { de combate que no pueden dirigirse contra un } \\
\text { objetivo militar concreto; o (c) en los que se } \\
\text { emplean métodos o medios de combate cuyos } \\
\text { efectos no sea posible limitar, como exige el } \\
\text { DIH; y que, en consecuencia, pueden alcanzar } \\
\text { indistintamente, en cualquiera de tales casos, } \\
\text { tanto a objetivos militares como a personas civiles } \\
\text { o bienes de carácter civil. Asimismo, la norma } 13 \\
\text { prevé de manera explícita que quedan prohibidos } \\
\text { los ataques por bombardeo, cualesquiera que } \\
\text { sean los métodos o medios utilizados (...). } \\
\text { De otro lado, las Normas } 70 \text { y } 71 \text { establecen } \\
\text { los principios generales sobre el empleo de las }\end{array}$ \\
\hline $\begin{array}{l}\text { Conflictos armados } \\
\text { no internacionales }\end{array}$ & $\begin{array}{l}\text { El Artículo } 13.2 \text { del Protocolo Adicional II (PAII) a los } \\
\text { Convenios de Ginebra de } 1949 \text { señala que no serán objeto } \\
\text { de ataque la población civil como tal, ni las personas civiles. } \\
\text { Asimismo, precisa que quedan prohibidos los actos o } \\
\text { amenazas de violencia cuya finalidad principal sea aterrorizar } \\
\text { a la población civil. }\end{array}$ & $\begin{array}{l}\text { armas precisando que se prohíben aquellas cuyos } \\
\text { efectos sean indiscriminados. } \\
\text { Posteriormente, las normas } 72 \text { a } 86 \text { prohíben } \\
\text { el uso de determinadas armas como balas } \\
\text { expansivas, balas explosivas, armas cuyo } \\
\text { efecto principal es lesionar mediante fragmentos } \\
\text { no localizables, armas trampa, y armas laser. } \\
\text { Asimismo, especifican el uso de determinadas } \\
\text { armas como minas terrestres. }\end{array}$ \\
\hline
\end{tabular}

Fuente: Elaboración propia basada en los Convenios de Ginebra de 1949, los Protocolos Adicionales de 1977 y las normas consuetudinarias del DIH

$\mathrm{Y}$ un tercer hito que es importante destacar incluye otras fuentes normativas más específicas que complementan el marco legal anteriormente enunciado y que determinan la prohibición de determinadas armas, así como obligaciones de los Estados para asistir y remediar a quienes hayan sufrido las consecuencias por su uso(1). Así, desde el siglo XIX se han emitido diversos instrumentos internacionales que tuvieron por finalidad limitar de manera más clara, pero sobre todo puntual, los medios de combate(2).

(1) Por ejemplo, la Convención de Ottawa sobre la Prohibición de minas antipersonales, prevé en su artículo 6.3 obligaciones para reparar a víctimas de minas. Veáse más en https://www.apminebanconvention.org/fileadmin/APMBC/other_languages/spanish/MBC/ MBC_convention_text/Convencion_d_Ottawa_Espanol.pdf.

(2) Para mayores referencias véase Declaración de San Petersburgo (1868) sobre Proyectiles explosivos con un peso inferior a 400 gramos; Declaración de La Haya (1899) sobre Balas que se expanden y se aplastan en el cuerpo humano; Reglamento de La Haya (1907) sobre Veneno y armas envenenadas; Protocolo de Ginebra (1925) sobre Armas químicas; Convención sobre la prohibición de las armas químicas (1993); Protocolo de Ginebra (1925) sobe armas biológicas; Convención sobre la prohibición de las armas biológicas (1972); Protocolo I (1980) a la Convención sobre Ciertas Armas Convencionales Armas que lesionan mediante fragmentos no localizables en el cuerpo humano mediante rayos X; Protocolo III (1980) a la Convención sobre Ciertas Armas Convencionales sobre Armas incendiarias; Protocolo IV (1995) a la Convención sobre Ciertas Armas Convencionales sobre armas láser cegadoras; Protocolo II, según fue enmendado en 1996, a la Convención sobre Ciertas Armas convencionales Minas, armas trampa y "otros artefactos"; Convención sobre la prohibición de las minas antipersonal (1997) sobre Minas antipersonal; Protocolo V (2003) a la Convención sobre Ciertas Armas Convencionales Restos explosivos de guerra; Convención sobre municiones en racimo (2008) Municiones en racimo, entre otras. 
Todo este basto marco jurídico y su evolución hasta llegar a un marco protector más específico procura establecer mínimos para las partes enfrentadas atendiendo a otro principio que es el de limitación en la elección de medios y métodos (véase infra punto 4). Solo a través de la adecuada implementación de estas normas se podrá garantizar la debida protección a la población civil y sus bienes, así como a elementos indispensables para su supervivencia, al patrimonio cultural y lugares de culto, a obras e instalaciones que contienen fuerzas peligrosas y, desde luego, al medio ambiente. De ahí la importancia de que el uso de un arma pueda llevarse acorde a los principios elementales del DIH: distinción, proporcionalidad y necesidad militar que identifiquen las partes.

\section{El potencial de destrucción de las armas nucleares}

Tras haberse revisado el marco general que limita el uso de medios en conflictos armados, corresponde entender los alcances de las armas nucleares. Como punto de partida, en un nivel técnico el uso de un arma nuclear implica la liberación de toda la energía contenida en el núcleo de un átomo. De acuerdo con el Centro para el control de armas y la no proliferación, un arma nuclear es "un dispositivo que libera rápidamente energía nuclear, ya sea a través de la fisión o una combinación de fisión y fusión" (2012).

Entonces, cuando uno de estos dispositivos explosiona se produce la liberación de energía nuclear, que es la energía contenida en el núcleo de un átomo. Las armas nucleares cuya fuerza explosiva resulta de reacciones de fisión se denominan comúnmente bombas atómicas, que son las bombas utilizadas en las ciudades de Hiroshima y Nagasaki, mientras que las que obtienen gran parte o la mayor parte de su energía en reacciones de fusión nuclear se denominan armas termonucleares o bombas de hidrógeno (Geneva Academy, 2014).

De un lado, se puede tener una aproximación al uso pacífico de armas nucleares, que es la vía autorizada en los distintos textos de los tratados sobre armas nucleares, y de otro, los fines más cuestionados que son los militares. En relación al primer punto, el uso de energía nuclear de manera adecuada contribuye en los procesos industriales, en la medicina, la agricultura, en la conservación de alimentos, en la minería, entre otros campos (Planas, 2010).

De otro lado, desde una perspectiva militar la posesión de armas nucleares es considerada como parte de la estrategia de seguridad de Estados quienes consideran que sus solas reservas o conservación constituyen un pilar de protección y estabilidad. Desde las ciencias políticas se señala que la capacidad que tengan los Estados para la adquisición, transferencia o desarrollo de armas nucleares permite sostener un statu quo a nivel internacional. De ahí que los sistemas de armas nucleares se prueben periódicamente y con frecuencia se realicen ejercicios nucleares para poner en práctica planes de ataque ofensivo contra posibles adversarios (Kristensen, 2015).

En ese orden de ideas, como señala Ginsberg (1979), un Estado mantiene una supremacía nuclear no solo cuando posee armamento, sino cuando tiene la voluntad para usar esa capacidad nuclear. De este modo, el hecho de que múltiples Estados se constituyan hoy como potencias nucleares por su alta su posesión armamento nuclear y los ensayos que realizan, perpetuaría, en el terreno geopolítico, las relaciones de poder militar que tienen respecto de otros Estados.

Sin embargo, estas consideraciones de índole geopolítico deben tener presente principalmente las consecuencias jurídicas que devienen de los efectos del uso de armas nucleares. Así, es importante tener en cuenta que una explosión nuclear es mucho más potente que los explosivos convencionales dado que emite cuatro tipos de energía: onda expansiva, luz intensa, calor y radiación (Centro para el control de armas y la no proliferación, 2012), por lo que, a diferencia de las primeras, su impacto es letal. Esto quiere decir que, es imposible asegurar que su uso atienda a satisfacer la necesidad militar de las partes en conflicto, sino que excede dichos propósitos. Quiere decir que los efectos de un ataque nuclear difícilmente se dirigirán exclusivamente contra objetivos militares. Por el contrario, la afectación impactará también población civil, sus bienes y el medio ambiente en que viven.

Esto ha quedado demostrado en dos situaciones históricamente conocidas: los eventos ocurridos en Hiroshima y Nagasaki en 1945, que pusieron en evidencia los efectos inmediatos y a largo plazo que tiene un arma nuclear. A modo de recuento histórico, Estados Unidos y Japón se enfrentaron durante cuatro años, desde 1941 hasta 1945, en la denominada "Guerra del Pacífico", un conflicto armado internacional en el marco de la segunda guerra mundial. El 26 de julio de 1945, Harry Truman, ex presidente de Estados Unidos, exigió la rendición de Japón, 
de lo contrario se realizaría un ataque que desembocaría "una destrucción rápida y absoluta” (Hernández, 2014). Frente a la negativa del gobierno japonés, el ex presidente de Estados Unidos ordenó que se desplieguen ambos ataques, el primero en Hiroshima el 6 de agosto y el segundo en Nagasaki el 9 de agosto del mismo año.

De acuerdo con lo relatado por delegados del CIRC, para 1950 se calculaba que un aproximado de 340,000 personas murieron inmediatamente en dichos ataques (2021). Sin embargo, a la fecha no hay un número preciso que dé cuenta de la totalidad de decesos. Sumado a la pérdida de miles de civiles, hubo otros impactos importantes que se prolongaron con el paso de los años, y persisten incluso en la actualidad. En relación al total de personas afectadas, algunas estadísticas señalan que hubo cerca de 557,478 que experimentaron los efectos posteriores del ataque nuclear (Chappell, 2020). Este número responde a los efectos que dejó la radiación, traducidos en personas que adquirieron una discapacidad y otros perjuicios que se prolongaron en las futuras generaciones.

Así, por ejemplo, quienes sobrevivieron a los ataques, los Hibakusha (o en español "los afectados por la bomba atómica"), han compartido experiencias asociadas a la pérdida de todo el grupo familiar, adquisición de discapacidades y efectos a largo plazo en relación al deterioro de su salud ${ }^{(3)}$. Se han registrado situaciones asociadas a alteraciones genéticas de niños recién nacidos, y tendencias a la adquisición de tumores malignos y otras formas de cáncer de pulmón, estómago, hígado, leucémica, cáncer intestinal y linfomas malignos que surgieron con el paso de los años (CICR, 2015). Todo ello exige poner al centro de la discusión las consecuencias para las víctimas de dichos ataques.

Entre otros impactos producidos por el uso de este tipo de armas, gran parte de los bienes de la población civil desaparecieron y muchos medios de transporte fueron arrojados a metros de distancia. Asimismo, la alta demanda de prestación de servicios médicos urgentes hizo que los hospitales colapsen por la falta de equipos y medicinas disponibles para una atención masiva, y con médicos y enfermeras enfrentando tipos de heridas totalmente nuevos para los que no había tratamiento (Bernard, 2015). Ello pone de manifiesto el enorme potencial de destrucción que genera el uso de un arma nuclear en personas y lugares especialmente protegidos.

Todo ello no solo fue cuestionable en el contexto de la segunda guerra mundial, sino que tiene una relevancia actual indiscutible pues, lejos de estar en un estadio de eliminación total de armas nucleares, a la fecha su fabricación, utilización en ensayos, y su adquisición sigue estando vigente. Lo paradójico es que inclusive Estados con suficiente arsenal nuclear como Estados Unidos, siguen liderando la tabla de potencias nucleares (FAS, 2021) pese a haber organizado y ejecutado los ataques nucleares en Japón.

A modo de ejemplo, de acuerdo con el Instituto Internacional de Estocolmo para la Investigación de la Paz, si bien a inicios del 2021 se registró una disminución de armas nucleares $^{(4)}$ (de 13,400 en 2020, a 13,080), actualmente la cantidad de armas nucleares desplegadas en fuerzas operativas aumentó a 3,825 en comparación a las 3,720 que había en el 2020. Estos datos comprueban que las armas nucleares siguen cobrando relevancia en el marco de la apelación a la seguridad nacional de Estados como Rusia y Estados Unidos, los cuales poseen en conjunto más del $90 \%$ de los arsenales nucleares mundiales y programas para modernizar e innovar en el uso de dichas armas (SIPRI, 2021).

En suma, la evidencia histórica del uso de un arma nuclear lleva a afirmar que los daños causados son de carácter indiscriminado (véase tabla supra). Todas estas consecuencias personales, medioambientales y materiales ponen en evidencia una contravención del principio de limitación en la elección de armas, y a normas cardinales del DIH relativas a la distinción, proporcionalidad y necesidad militar, que deben considerarse de manera previa a cualquier ataque.

\section{El camino hacia la prohibición total de las armas nucleares}

Desde la primera vez que se emplearon las armas nucleares, la comunidad internacional ha venido realizando esfuerzos para promover el desarme y prohibir su uso. Así, en 1946, un primer gran hito fue la aprobación de una resolución de Asamblea General de las Naciones Unidas mediante la cual se

(3) Para revisar más testimonios véase https://www.atomicarchive.com/resources/documents/hibakusha/index.html.

(4) Esta cantidad representa el total de armas nucleares que poseen conjuntamente los nueve países que poseen armamento nuclear, Ios cuales son Estados Unidos, Rusia, Reino Unido, Francia, China, India, Pakistán, Israel y Corea del Norte. 
constituyó una Comisión encargada de estudiar los problemas surgidos con motivo del descubrimiento de la energía atómica ${ }^{(5)}$. Sumado a ello, con el paso de los años, distintas iniciativas a nivel internacional fueron cristalizando la necesidad de limitar el uso de dichas armas hasta llegar a prohibirlas en la actualidad.

Dentro de los instrumentos convencionales que regulan el uso de armas nucleares de manera específica se encuentran, de manera cronológica, el Tratado de Prohibición Parcial de los Ensayos Nucleares (en adelante, “TPPE”) de 1963; el Tratado para la Proscripción de las Armas Nucleares en América Latina y el Caribe (en adelante, "Tratado de Tlatelolco") de 1967; el Tratado sobre la No Proliferación de las Armas Nucleares (en adelante, "TNP") de 1968; el Tratado de Prohibición Completa de los Ensayos Nucleares (en adelante, "TPCE") de 1996; y el más importante por su carácter definitivo de prohibición, el Tratado sobre la Prohibición de Armas Nucleares (en adelante, "TPAN") de 2017. A continuación, se discutirán sus alcances y los principales desafíos en torno a su efectividad.

Desde 1960 los tratados que versaban sobre armas nucleares tendían más bien hacia la prohibición de pruebas o ensayos de armas nucleares en la atmósfera, en el espacio ultraterrestre y bajo el agua, y propugnaba la creación de zonas libres de armas nucleares, en donde no se realicen pruebas que podrían tener cierto impacto en el espacio geográfico. El primero de los tratados, el TPPE, contenía todas las disposiciones antes mencionadas y entró en vigor el 10 de octubre de 1963. A la fecha ha sido ratificado por 125 Estados alrededor del mundo.

En la misma línea que el TPPE, en 1967 se negoció un tratado trascendental en la región americana, el Tratado de Tlatelolco. El principal aporte jurídico de este instrumento fue apostar por la consolidación de una tendencia hacia la prohibición del desarrollo, adquisición, y traspaso directa o indirectamente en América Latina y el Caribe. Los orígenes de este instrumento se remontan a la Guerra Fría, especialmente al conflicto ocurrido entre Estados Unidos, la Unión de Repúblicas Socialistas Soviéticas y Cuba en 1962, conocido como la crisis de los misiles de Cuba. Como señala Martínez, la sospecha de una la instalación de dispositivos nucleares en Cuba dio paso a creer que América Latina debía ser una zona libre de armas nucleares y que una resolución para desnuclearizar la región disminuiría la tensión generada por aquel conflicto (1982).

De este modo, México lideró los esfuerzos en convertir América Latina en una zona libre de ensayos nucleares, lo que incluyó el compromiso de no fabricar, recibir, almacenar ni ensayar armas nucleares 0 artefactos de lanzamiento nuclear (OPANAL, 1987). Entonces, la importancia de este instrumento fue la creación de la primera zona libre de armas nucleares del mundo en los Estados de América Latina y el Caribe. Asimismo, se acordó que el uso exclusivo de la energía nuclear se daría solo para fines pacíficos en aras de su desarrollo económico y progreso social (art. 17). A la fecha, 33 Estados han firmado y ratificado el Tratado de Tlatelolco.

Otro gran hito se da al año siguiente, en 1968, con la aprobación del TNP, cuya naturaleza principal fue dar un espaldarazo al objetivo de desarme nuclear general y completo, y así prevenir la propagación y desarrollo de armas nucleares, tecnología y/o piezas armamentísticas. De igual manera, el compromiso de no proliferación incluyó no generar programas nucleares cuya finalidad sea militar, sino solamente con fines pacíficos, y así dotar de contenido al principio de mantenimiento de la paz y la seguridad internacionales dispuesta en la Carta de Naciones Unidas.

Este tratado entró en vigor el 5 de marzo de 1970 y ha sido ratificado por 191 Estados. Sin embargo, lamentablemente entre los Estados que no han ratificado el tratado se encuentran aquellos que tienen reservas nucleares tales como India, Israel y Pakistán ${ }^{(6)}$. Pese a la importancia de este tratado para garantizar la agenda de desarme, el cumplimiento de las finalidades del TNP se ha visto seriamente cuestionada por programas nucleares clandestinos sostenidos por algunos Estados, tales como Libia, Irán y Corea del Norte, lo que "puso en crisis la confianza internacional en la efectividad del Tratado como piedra angular del esfuerzo en contra de la proliferación" (Andereya, 2008).

Otro tratado importante en relación al ejercicio de ensayos nucleares se dio en

(5) Véase más en Asamblea General de Naciones Unidas. (1946). Resoluciones aprobadas de acuerdo con los informes del Primer Comité. https://www.un.org/ga/search/view_doc.asp?symbol=A/RES/1(I)\&Lang=S.

(6) Véase más en: Arms Control Association. (s. f.). Israel, India, and Pakistan: Engaging the Non-NPT States in the Nonproliferation Regime. Arms Control Today. https://www.armscontrol.org/act/2003-12/features/israel-india-pakistan-engaging-non-npt-statesnonproliferation-regime\#topofpage. 
1996, fecha en que se aprobó el TPCE. El principal aporte de este tratado fue la prohibición de llevar a cabo explosiones de ensayo de armas nucleares en la jurisdicción de los Estados Parte. Desafortunadamente, los esfuerzos de negociación no han podido ser implementados pues si bien fue firmado por 185 Estados y ratificado por 170, a la fecha aún no entra en vigor. Esto se debe a que, dentro de sus provisiones se previó que el tratado entraría en vigor cuando fuese ratificado por 44 Estados específicos que son parte de la Conferencia sobre desarme. A la fecha 8 de esos Estados aún no lo han ratificado, a saber, China, India, Pakistán, Corea del Norte, Israel, Irán, Egipto, y Estados Unidos ${ }^{(7)}$. Este escenario dificulta la implementación y eficacia de este texto jurídico.

La entrada en vigor de este tratado es de alta importancia debido a las importantes consecuencias que tienen los ensayos nucleares no solo en la salud de quienes se ven expuestos, sino principalmente del medio ambiente. Como señala la Organización del Tratado de Prohibición Completa de los Ensayos Nucleares (CTBTO por sus siglas en inglés), los ensayos provocan la dispersión de material radioactivo que, dependiendo del tipo de ensayo, podrá darse en la atmósfera, superficie terrestre o en el agua, lo que en última instancia puede perjudicar el consumo humano.

Finalmente, el último de los tratados y el más importante por sus alcances es el TPAN que se erige como el primer acuerdo de carácter universal que prohíbe las armas nucleares por completo. Su naturaleza, al igual que el TNP está orientada al desarme, por lo que se puede afirmar que ambos tratados se complementan. El texto de este tratado fue aprobado en 2017 en el marco de la Conferencia de las Naciones Unidas para negociar un tratado de prohibición de las armas nucleares, y entró en vigor en el 22 de enero de 2021. Actualmente 56 Estados son Parte en este Tratado, lamentablemente al igual que en tratados anteriores, aquellos Estados con mayor arsenal nuclear no han ratificado estas obligaciones: Estados Unidos, Rusia, Israel e India, por mencionar algunos ejemplos.

En línea con los textos de tratados anteriores, el TPAN compromete a los Estados a "nunca y bajo ninguna circunstancia", desarrollar, ensayar, producir, fabricar, adquirir, trasferir, poseer, usar o amenazar ni almacenar armas nucleares $u$ otros dispositivos explosivos nucleares (art. 1). Igualmente, importante es que prevé un deber de cooperación para que aquellos Estados que tuviesen armas después de julio de 2017, a efectos de verificar la eliminación irreversible de su programa de armas nucleares (art. 4.1). Y, en línea con los tratados previos salvaguarda el "derecho inalienable" de los Estados Parte a desarrollar la energía nuclear con fines pacíficos. Un punto para destacar es que, a diferencia de los tratados anteriores, contiene especificaciones para asistir a las víctimas de desastres nucleares sin discriminación y de acuerdo a sus necesidades específicas (art. 6.1).

La evolución normativa de la prohibición de ensayos y uso de armas nucleares responde al peligro que supondría una nueva detonación, así como al compromiso de la comunidad internacional para no repetir sucesos como los ocurridos en 1946. Desafortunadamente, los Estados que más utilizan armas nucleares no son todavía Parte en la mayoría de estos tratados, lo que vuelve a poner en entredicho la voluntad para evitar su utilización.

\section{Las armas nucleares bajo la lupa del DIH: Un análisis jurídico de los principios}

A efectos de analizar si el uso de un arma nuclear se alinea a lo señalado en el DIH, específicamente en relación a las normas sobre medios y métodos mencionadas en el primer punto (véase cuadro supra), es importante retomar un principio transversal a toda la conducción de hostilidades que es el de limitación. Según el DIH, las partes en conflicto no tienen libertad irrestricta para optar por los medios que utilizarán en el campo de batalla. De hecho, algunos instrumentos convencionales del DIH como las Reglas de La Haya de 1899 y 1907, disponen que "los beligerantes no tienen un derecho ilimitado en cuanto a la elección de medios para dañar al enemigo" (art. 22). Para entender, entonces, qué armas se pueden utilizar, debe analizarse cuáles son los efectos que estas tienen.

Algunos pronunciamientos importantes que dan luces sobre la legalidad o no del uso de armas nucleares se remontan a 1996 cuando la ClJ emitió una opinión

(7) Véase más en Naciones Unidas. (2021, 27 de setiembre). Tratado de Prohibición de Pruebas Nucleares: 25 años a la búsqueda de un mundo sin libre de armas atómicas. https://news.un.org/es/story/2021/09/1497532. 
consultiva sobre la legalidad de la amenaza o el empleo de armas nucleares. Otro pronunciamiento importante es el posicionamiento del CICR como organismo humanitario cuyo manato radica en el cumplimiento de las normas del DIH en situaciones de conflicto armado. Teniendo en cuenta ambos desarrollos jurídicos, se conducirá un análisis sobre los principios cardinales del DIH: distinción, proporcionalidad y necesidad militar, que permitirá arribar a la conclusión sobre si la amenaza o uso se encuentra justificado en el plano jurídico.

Como primer aspecto, en el año 1994, algunos Estados no poseedores de armas nucleares, impulsaron a través de la Asamblea General de Naciones Unidas el pedido para que la Corte que se pronuncie en el marco de su competencia consultiva, sobre la amenaza o el uso de armas nucleares. Es así como en diciembre de 1994 la Asamblea General mediante su resolución A/RES/49/75 aprobó la solicitud de una opinión consultiva a la $\mathrm{ClJ}$ de modo que respondiera, con urgencia, pregunta: "¿La amenaza o el uso de armas nucleares en alguna circunstancia está permitida por el Derecho internacional?"(8). En respuesta a ello, la CIJ en 1996 emitió su opinión consultiva.

Aunque parezca evidente, una de las primeras cuestiones que destacó la Corte es que no cabía duda de la aplicabilidad del DIH al uso de armas nucleares. Esta pertinente aclaración respondió a la apelación de algunos Estados sobre su inaplicación debido a la aparición de las armas nucleares con posterioridad a la existencia de la mayoría de los principios y normas del DIH. En esa línea, la Corte recordó que el poder destructivo de las armas nucleares no se puede contener ni en el espacio ni en el tiempo (párr. 35) y precisó que, aunque no haya una prohibición específica, su legalidad debe cuestionarse a partir del análisis de normas y principios del DIH (párr. 86). Es decir, se puede llevar a cabo un pronunciamiento sobre el fondo del asunto con el marco jurídico general del DIH (véase cuadro supra y punto 3 ).

Ahora bien, a pesar de ello la Corte no concluyó su naturaleza como arma prohibida pero sí estableció, o más bien recordó, que existe una consideración primordial que es el respeto irrestricto del principio de humanidad. Al respecto, estableció que la conducción de hostilidades debe encontrarse supeditada a una serie de requisitos estrictos, que tienen que ver con la exclusión de cualquier arma que no distinga entre población civil y objetivos militares, o que pueda dar lugar a sufrimientos innecesarios a quienes participan de estas (párr. 95). Por lo tanto, aunque la CIJ no haya señalado categóricamente que las armas nucleares son ilegales, sí anticipó que el análisis jurídico debía ceñirse al marco normativo establecido en el DIH.
Aunque para muchos académicos como Greenwood se habría desaprovechado una oportunidad histórica de declarar que el uso de las armas nucleares es ilícito en todas las circunstancias (1997), los alcances de la opinión consultiva de la Corte legitiman el respeto de las normas del DIH, y a su aplicación a las nuevas armas. De este modo, se marca un hito importante en la determinación del uso de los medios y métodos aplicables a situaciones de conflicto armado.

Como segundo punto, los comentarios y posicionamiento jurídico del $\mathrm{CICR}$ expresados en el cuarto Reporte del CICR sobre "el DIH y los desafíos de los conflictos armados contemporáneos" reafirman la posición de la $\mathrm{ClJ}$ ahondando en un análisis técnico/ jurídico sobre su uso, y concluyendo que este sí tendría un impacto a nivel legal. Así, tras un análisis de compatibilidad con los principios del DIH, se concluye que la utilización de armas nucleares en cualquier circunstancia sería catastrófica ya que, por su naturaleza y efectos, son incapaces de cumplir el primero de los requisitos en toda situación de conflicto armado: la distinción entre objetivos militares y quienes no participan de manera directa en el enfrentamiento.

En la misma línea, destaca el carácter indiscriminado que genera un ataque nuclear pues por la naturaleza del diseño técnico del arma su principal efecto es dispersar el calor por radiación, lo que ocurriría solo mediante una explosión. $Y$ esto último, en la mayoría de los escenarios y, tal como ha mostrado el curso de la historia, impactaría en áreas geográficas altamente concentradas y extensas. Además de ello, el calor generado por la explosión provocaría lesiones graves como quemaduras en la piel u otras graves consecuencias como las ya expuestas en el marco del conflicto armado internacional entre Estados Unidos y Japón.

De hecho, el impacto del arma, además de las tasas de letalidad, tiene consecuencias para quienes se encuentren incluso a tres kilómetros del epicentro, sin considerar

(8) Para mayores referencias véase Asamblea General de Naciones Unidas. (1994). Resolución A/RES/49/75 (p.17). https://undocs. org/pdf?symbol=es/A/RES/49/75. Asimismo, véase Corte Internacional de Justicia. (1994). Solicitud de opinión consultiva. https:// www.icj-cij.org/public/files/case-related/95/7646.pdf. 
también la enorme destrucción de edificios e infraestructura a varios kilómetros (CICR, 2015). Todo ello, nuevamente, evidenciaría la falta diligencia al definir los objetivos militares que, por su naturaleza normativa deben generar una ventaja militar concreta y directa, que es aquella que se prevé del ataque militar considerado en su conjunto y no se analiza de manera aislada o particular ${ }^{(9)}$.

Un aspecto final que rescata el mencionado Reporte es el de la respuesta a los ataques realizados. Es decir, existen altas probabilidades de que lo que ocurra tras el uso de un arma nuclear, sea una escalada de ataques que impliquen un uso aún mayor de armas nucleares por las partes en conflicto, con consecuencias humanitarias catastróficas (CICR, 2015). Sin embargo, incluso en este escenario de ius ad bellum en donde los Estados pueden ejercer su inmanente derecho a la legítima defensa, conforme a la Carta de Naciones Unidas (art. 51), estos no se encuentran eximidos de respetar las normas del DIH que incluyen la limitación en el uso de medios.

Ahora bien, con todo el desarrollo normativo existente, así como los pronunciamientos jurídicos y técnicos que ha tenido el debate sobre el uso de armas nucleares, el último punto a destacar en esta sección es el paso a paso en el examen jurídico para terminar de comprender si el uso de armas nucleares cumple o no con los estándares normados en el DIH. Sobre el particular, se desarrollarán los principios de distinción, proporcionalidad y necesidad militar.

De manera preliminar, resulta importante aclarar que no se ha optado por discutir los alcances del principio de precaución en el uso de armas nucleares debido a que la explosión de un arma nuclear impide desplegar todo el cuidado factible y constante de cara a preservar tanto como se pueda a los civiles y los bienes de carácter civil, conforme dictamina este principio $^{(10)}$. A mayor abundamiento, ¿cómo podrían adoptarse todas las medidas de precaución factibles para minimizar los sufrimientos y daños a civiles, y al medio ambiente? Incluso, si se tiene como ejemplo uno de los Manuales militares de Estados Unidos que relega la precaución al análisis de las condiciones operativas (2019)(11), se sabrá que no hay posibilidad de respetar el principio de precaución con el uso de un arma cuyo impacto tiene niveles incuantificables de destrucción incluso desde el terreno operativo.
En relación a la distinción(12) se erige como un llamado de atención a las partes en conflicto para que los ataques se dirijan exclusivamente hacia objetivos militares y no contra población civil o personas que están fuera de combate (hors de combat). La importancia de este principio radica en brindar la protección más amplia a quienes lejos de participar en las hostilidades son gran parte de las veces quienes sufren las mayores consecuencias. En esa línea, la precitada opinión consultiva de la CIJ afirmó que el principio de distinción era uno de los "principios cardinales" del DIH y uno de los "principios intransgredibles" del Derecho Internacional Consuetudinario (1996). Asimismo, de esta regla se desprende que, en áreas donde civiles y bienes de carácter civil se mezclan con combatientes y objetivos militares, las partes debe hacer todo lo posible para verificar que los blancos de ataque sean objetivos militares (Maresca \& Mitchell, 2015)

Sobre el particular, para afirmar si se distingue o no a quienes participan de las hostilidades conviene formular la pregunta sobre si el Estado puede o no controlar el efecto de un arma nuclear. El curso de la historia ha demostrado que la explosión de estos dispositivos es inmanejable en cuanto a su impacto. En esa medida, si no se puede tener el control sobre el impacto del arma, no se cumpliría con el objetivo básico de la distinción que es discriminar entre quienes participan directamente de las hostilidades y pueden constituirse como un objetivo militar, de quienes no. $Y$ en este punto, es relevante precisar que un objetivo militar es aquel que por su naturaleza, ubicación, finalidad o utilización contribuye de manera eficaz a la acción militar, y cuya destrucción total o parcial, captura o neutralización, ofrecerá una ventaja militar concreta y directa ${ }^{(13)}$. Como se

(9) Véase más en la práctica de Estados como Australia, Bélgica, Canada, Francia, Alemania, Itala, Holanda, Nueza Zelanda, Nigeria, España, Reino Unido y Estados Unidos. Compilado de IHL database. https://ihl-databases.icrc.org/customary-ihl/eng/docs/v1_rul_ rule14\#Fn_A9C8FAD0_00027.

(10) Véase más en Artículo 57 del Protocolo Adicional I, y Norma 15 y 16 del DIH consuetudinario.

(11) El Manual Militar señala que el "adoptar precauciones que sean factibles depende en gran medida del contexto, incluidas las consideraciones operativas".

(12) El principio de distinción está codificado en los artículos 48, 51 (2) y 52 (2) del Protocolo adicional I, y en el artículo 13 (2) del Protocolo adicional II. También forma parte del DIH consuetudinario, consagrado en la norma 1.

(13) Véase más en Protocolo Adicional I, art. 52.2 y norma consuetudinaria del DIH 8. 
puede evidenciar de los hechos históricos, un arma nuclear no proporciona una ventaja militar al eliminar uno de los objetivos militares, por el contrario, su explosión conlleva bajas militares, civiles, y destrucción material y medioambiental generalizada.

Pero si incluso, como señaló el Juez Schwebel de la ClJ, se pudiese adaptar el uso de un arma nuclear a las dimensiones necesarias y técnicas que favorezcan la satisfacción del principio de distinción, como el uso de "armas nucleares tácticas contra submarinos que están equipados con armas nucleares como objetivos militares o navales discretos"(14), incluso así se tendría que analizar el efecto que estas tienen. $Y$ estos, en cualquier circunstancia, siguen siendo controvertidos porque conllevan la contravención de la norma que prohíbe daños superfluos o sufrimientos innecesarios (véase cuadro supra). Entonces, si incluso el arma llegase a diferenciar, es menester tener presente sus efectos en el objetivo militar.

Con respecto a la proporcionalidad ${ }^{(15)}$, el uso de armas nucleares tampoco superaría este análisis. Esto es, que el objetivo militar que se busque atacar proporcione una ventaja militar concreta y directa mayor a los daños que pueda causar. Como señala Salmón:

Cuando son atacados objetivos militares, las personas civiles y los bienes de carácter civil deben preservarse lo más posible de daños incidentales y estos además no deben ser excesivos con respecto a la ventaja militar esperada de cualquier ataque contra un objetivo militar". En el empleo de armas nucleares no habría dicha proporción, por el contrario, la regla seria la presencia de daños incidentales y la excepción el ataque exclusivo hacia un objetivo militar. Dicho en otras palabras, las personas seguirían siendo las principales afectadas con los efectos indiscriminados de la radiación (2015).

Hasta aquí se ha abordado la situación de la proporcionalidad en relación a dos de los bienes jurídico más importantes: la vida y la dignidad del individuo. Sin embargo, es crucial añadir los otros daños que dejaría un ataque nuclear, en particular en lo tocante al respeto del medio ambiente. En ese orden de ideas, como sostiene la Organización Mundial de la Salud la radiación emanada de un arma nuclear es altamente nociva para los cultivos y la cadena alimentaria, así como para el ganado, pues contamina los productos que se extraen de este (1993). Sus efectos negativos también se expanden en el ecosistema marino, provocando que las poblaciones se encuentren en una situación de vulnerabilidad mayor frente al cambio climático.
De manera general, el DIH prevé también disposiciones elementales de protección al medio ambiente, que señalan que quedan prohibidos aquellos métodos o medios de guerra que causen daños extensos, duraderos y graves al medio ambiente natural ${ }^{(16)}$. Esta disposición recoge un estándar alto pues exige que conjuntivamente concurran estas tres características para entender que un arma estaría prohibida.

Sin embargo, existe también otra convención que establece un estándar más bajo, pero que permite una protección mucho mayor del medio ambiente en contextos de conflicto armado. Así, la Convención sobre la prohibición del uso de técnicas de modificación del medio ambiente con fines militares u otros fines hostiles (ENMOD por sus siglas en inglés) de 1976 establece una disposición similar a la mencionada del Protocolo Adicional I, pero con una diferencia significativa. Concretamente prohíbe a los Estados modificar el ambiente con técnicas que tengan un efecto extenso, duradero o grave en este (art. 1); así, la técnica utilizada en este tratado apuesta por la disyunción entre estas tres características empleando el término "o", que permite caracterizar la violación de dicha disposición con la constatación de cualquiera de los tres efectos.

Finalmente, se debe analizar el principio de necesidad militar. Este principio tiene especial importancia porque su uso irrestricto podría llevar, y en la práctica ha llevado, a justificar situaciones de gran abuso y excesos durante conflictos armados, como ocurrió en la Segunda Guerra Mundial. En ese sentido, la necesidad militar, que aparece por primera vez en el Código de Lieber ${ }^{(17)}$ se estableció con miras a adoptar aquellas medidas que son indispensables para asegurar el fin de la guerra y que son lícitas según las leyes y usos modernos de la guerra (1863, art. 14).

(14) Véase más en Opinión disidente del Juez Schwebel sobre la Opinión Consultiva sobre legalidad de la amenaza o el empleo de armas nucleares (1996). https://www.icj-cij.org/public/files/case-related/95/095-19960708-ADV-01-09-EN.pdf.

(15) El principio de proporcionalidad en el ataque está codificado en el artículo 51 (5) (b) del Protocolo adicional l, y repetido en el artículo 57. De igual manera está contenido en la Norma 14 del DIH consuetudinario.

(16) Esta disposición está recogida en el artículo 35.3 del Protocolo Adicional I.

(17) Este código era uno de corte militar entregado a personal de las fuerzas armadas de Estados Unidos con instrucciones sobre la conducción de hostilidades en el campo de batalla. Véase más alcances de este principio en los artículos 15 y 16. 
De ahí que como señala Schmitt la necesidad militar deba coexistir en equilibrio simbiótico con el principio de humanidad, que busca limitar el sufrimiento y la destrucción del incidente de la guerra (2010). Sin embargo, el despliegue de arsenal nuclear en contextos de conflicto armado no atiende al principio de necesidad militar pues no tiene como objetivo el sometimiento de la parte adversa, sino que sus efectos destructivos son mucho más amplios dado que exceden los objetivos propios del enfrentamiento.

A modo de síntesis, el uso de un arma nuclear sea que pueda adaptarse a la distinción o no, genera daños y sufrimientos innecesarios con el impacto que tiene. De ahí que no supere los estándares normativos elementales a considerar en situaciones de conflicto armado, y que por lo tanto su uso en ensayos o potencialmente en conflictos armados resulte contraria a Derecho. A mayor desarrollo, su uso no solo conlleva una grave violación al DIH, sino que también constituiría un posible crimen de guerra debido al uso de armas cuyos efectos son indiscriminados. Esta caracterización penal se encuentra bajo los alcances del Estatuto de Roma de la Corte Penal Internacional, espacio en donde se determina la responsabilidad penal individual de quien comete este tipo de crímenes.

\section{Conclusiones: ¿Estamos frente a una tendencia hacia el desarme nuclear?}

Actualmente la prohibición del ensayo amenaza y/o de uso de armas nucleares viene siendo discutida tanto en la apelación al uso de la fuerza de manera legítima o ius ad bellum, como en la propia conducción de hostilidades que se da en el marco del ius in bello. De esta manera, como se ha revisado, distintos instrumentos convencionales se han impulsado para comprometer a los Estados a tomar parte activa en una agenda hacia la no proliferación y el desarme, y que se eviten episodios como los ocurridos en Hiroshima y Nagasaki. Sin embargo, la falta de ratificación de estos tratados por parte muchos Estados, principalmente de aquellos que poseen arsenal nuclear, así como la ejecución de ensayos nucleares evidencian un problema aún latente: la posibilidad de su utilización en situaciones de conflicto armado.

Como se ha ido analizando, su uso potencial conllevaría una serie de riesgos personales, medioambientales y materiales para quienes no toman parte activa en las hostilidades, y en un plano jurídico, un eventual incumplimiento de las obligaciones impuestas en el DIH, así como la comisión de crímenes de guerra. Esto sucede principalmente porque el DIH se conduce bajo la premisa de que las partes enfrentadas no tienen una posibilidad ilimitada de decidir qué medios o armas utilizar, sino que su elección debe responder a ciertas normas que se sustentan, a su vez, en el principio de humanidad.

Para concluir si el uso de un arma nuclear es lícito o no a la luz del DIH, es imprescindible evaluar si supera el examen de distinción, proporcionalidad y necesidad militar. Esto en términos generales supone cuestionar si el impacto del arma (i) diferenciará entre objetivos militares legítimos en el DIH y población civil y sus bienes; (ii) si sus efectos serán indiscriminados o no; y (iii) si su uso responde a una ventaja militar concreta y directa, o por el contrario traerá mayores consecuencias en relación al objetivo de someter a la parte adversa. Al haber revisado cada una de manera detallada es imposible concluir su legalidad. Inclusive en aquel supuesto hipotético en que la tecnología llega a adaptar el arma para poder superar el examen de distinción, sus efectos seguirán siendo los mismos que en 1946 pues causarán daños superfluos y sufrimientos innecesarios, y además prolongados en el tiempo, a quienes alcance.

De ahí que un primer gran desafío radique en el impulso de compromisos serios para evitar la amenaza de su uso en situaciones de conflictos armados, y dar pasos firmes hacia el desarme y el impedimento de su utilización en ensayos nucleares. Un segundo reto que posa la tenencia y distribución de armas nucleares es el desarrollo de programas nucleares con miras hacia la utilización de tecnología para su potencial utilización. Ello aletarga y estanca las negociaciones, como ha quedado evidenciado con la falta de entrada en vigor del TPCE.

Finalmente, no es un asunto menor que las consecuencias nocivas del empleo de armas nucleares deben leerse a partir de los sucesos históricos como los ocurridos en Hiroshima y Nagasaki. Ambos han puesto en evidencia las consecuencias de no revisar escrupulosamente los medios a utilizar en situaciones de conflicto armado y no considerar como eje de la conducción de hostilidades al principio de humanidad. Ante ello, desde el plano jurídico el incumplimiento entraña consecuencias que pueden devenir en la determinación de responsabilidad internacional de los Estados, así como de la 
responsabilidad penal individual. Por todo ello, resulta vital que los Estados que son potencia nuclear asuman un compromiso contundente hacia la eliminación de armas y ensayos nucleares, el cual solo se verá cristalizado con la ratificación de los tratados en cuestión y un mecanismo de seguimiento y rendición de cuentas que asegure un cumplimiento cabal del mismo.

\section{Referencias bibliográficas}

Andereya, A. (2008). La crisis de credibilidad del tratado sobre la no proliferación de las armas nucleares. Revista Diplomacia, 117. https:// www.corteidh.or.cr/tablas/r22604.pdf

Aragón, A. (2017). Lógica y naturaleza de los retos al sistema de desarme y no proliferación nuclear en el siglo XXI. Revista de Relaciones Internacionales de la UNAM, 129. http://revistas.unam. $\mathrm{mx} /$ index.php/rri/article/view/63230

Bernard, V. (2015). Un precio demasiado alto: repensar las armas nucleares a la luz de su costo humano. Revista Internacional de la Cruz Roja, 97(899), 499-506. https://www.cambridge.org/core/journals/ international-review-of-the-red-cross/article/price-too-high-rethinkingnuclear-weapons-in-light-of-their-human-cost/2ABEF30BF8AA544F7 FD78F39A2847E9D\#fn2

Centers for Disease Control and Prevention (CDC). (2012). Armas Nucleares. https://www.cdc.gov/nceh/radiation/emergencies/pdf/ infographics/infographic_nuclear_weapon_es.pdf

Chappell, E. (2020). Hibakusha Memories: Between the Generations. Wasafiri, 35(2). https://doi.org/10.1080/02690055.2020.1721143

Comité Internacional de la Cruz Roja (CICR). (2015). 32nd Internacional Conference of the Red Cross and Red Crescent. International humanitarian law and the challenges of contemporary armed conflicts.

Comité Internacional de la Cruz Roja (CICR). (2015). Consecuencias de largo plazo de las armas nucleares en la salud. Nota informativa 5. https://www.icrc.org/es/download/file/10635/consecuencias_de_ largo_plazo.pdf

Comité Internacional de la Cruz Roja (CICR). (2021). Tratado sobre la Prohibición de las Armas Nucleares. https://www.icrc.org/es/document/ tratado-sobre-la-prohibicion-de-las-armas-nucleares-de-2017

Consejo de Seguridad Nuclear. (s.f). La energía nuclear. https://www. csn.es/la-energia-nuclear

Federation of American Scientists. (2021). Status of World Nuclear Forces. https://fas.org/issues/nuclear-weapons/status-world-nuclearforces/

Fujita, H. (1997). Sobre la Opinión consultiva de la Corte Internacional de Justicia acerca de la licitud de las armas nucleares. Revista Internacional de la Cruz Roja, 22(139), 59-68. https://www.icrc.org/ es/doc/resources/documents/misc/5tdldg.htm

Geneva Academy. (2014). Nuclear Weapons Under International Law. https://www.geneva-academy.ch/joomlatools-files/docman-files/ Nuclear\%20Weapons\%20Under\%20International\%20Law.pdf

Ginsberg, S. (1979). Función del poder nuclear en la política internacional. Revista de Marina. https://revistamarina.cl/ revistas/1979/6/ginsberg.pdf
Greenwood, C. (1997). Opinión consultiva sobre las armas nucleares y la contribución de la Corte Internacional de Justicia al derecho internacional humanitario. Revista Internacional de la Cruz Roja, 22(139), 69-80. https://www.icrc.org/es/doc/ resources/documents/misc/5tdlb3.htm

Hernández, S. (2014). Migración, comercio y guerra: las relaciones entre Japón, México y Estados Unidos antes de Pearl Harbor. México y la Cuenca del Pacífico, 6, 103-138. https://www. redalyc.org/pdf/4337/433747296006.pdf

International Court of Justice. (1949). The Corfu Channel case. https://www.icj-cij.org/public/files/ case-related/1/001-19490409-JUD-01-00-EN.pdf

International Court of Justice. (1996). Advisory Opinion on the Legality of the Threat or Use of nuclear weapons. https://www.icj-cij.org/public/ files/advisory-opinions/advisory-opinions-1996es.pdf

Junod, M. (1982). El desastre de Hiroshima (I). Revista Internacional de la Cruz Roja, 7(53), 289305. https://www.icrc.org/es/doc/assets/files/other/ irrc-230-junod.pdf

Kristensen, H. \& Mckinzie, M. (2015). Nuclear arsenals: Current developments, trends and capabilities. International Review of the Red Cross. https://international-review.icrc.org/sites/default/ files/irc97_3_web-reduced.compressed.pdf

Lebherz, T. \& Ellinger, F. (1963). Las radiaciones y el embarazo. Medical Annals of the District of Columbia, 140-144. https://iris.paho.org/ bitstream/handle/10665.2/15203/v54n2p140. pdf?sequence=1\&isAllowed $=y$

Maresca, L. \& Eleanor, M. (2015). The human costs and legal consequences of nuclear weapons under international humanitarian law. International Review of the Red Cross. https://internationalreview.icrc.org/sites/default/files/irc97_3_webreduced.compressed.pdf

Martínez, J. (1982). Zona libre de armas nucleares en la América Latina. IAEA Bulletin, 24(2), 56-58. https://www.iaea.org/sites/default/ files/24203595658_es.pdf

Organismo para la Proscripción de las Armas Nucleares en la América Latina y el Caribe. (s. f). Zonas Libres de Armas Nucleares (ZLAN). https://www.opanal.org/zonas-libres-de-armasnucleares-zlan/

Organismo para la Proscripción de las Armas Nucleares en la América Latina y el Caribe. (1967). Tratado de Tlatelolco. https://www.opanal.org/ tratado-de-tlatelolco/

Organismo para la Proscripción de las Armas Nucleares en la América Latina y el Caribe. (1987). 
Vigésimo aniversario del tratado de Tlatelolco, 1967 - 1987.

Organización de las Naciones Unidas. (s. f.). Armas Nucleares. https://www.un.org/disarmament/es/ adm/nuclear-weapons/

Organización de las Naciones Unidas. (1945, 26 de junio). Carta de Naciones Unidas. https://www. un.org/es/about-us/un-charter

Organización de las Naciones Unidas. (1963). Tratado sobre la prohibición de ensayos nucleares. https://treaties.unoda.org/t/test_ban

Organización de las Naciones Unidas. (1968). Tratado sobre la No Proliferación de las Armas Nucleares. https://treaties.unoda.org/t/npt

Organización de las Naciones Unidas. (1996). Tratado de Prohibición Completa de los Ensayos Nucleares. https://treaties.unoda.org/t/ctbt

Organización de las Naciones Unidas. (2020). Conferencia de las Partes de 2020 encargada del examen del Tratado sobre la No Proliferación de las Armas Nucleares (TNP). https://www.un.org/ es/conferences/npt2020
Organización Mundial de la Salud. (1993). Efectos de las armas nucleares en la salud y el medio ambiente. http://apps.who. int/iris/bitstream/handle/10665/202932/WHA46_30_spa pdf;jsessionid=16E7B707A2EBC18F374F451C7742E874?sequence $=1$

Salmón, E. (2015). Introducción al Derecho Internacional Humanitario. Comité Internacional de la Cruz Roja. https://idehpucp.pucp.edu. pe/wp-content/uploads/2012/10/Introducci\%C3\%B3n-al-DerechoInternacional-Humanitario-2012-3.pdf

Schmitt, M. (2010). Military Necessity and Humanity in International Humanitarian Law: Preserving the Delicate Balance. Virginia Journal of International Law, 50(4), 795-839.

Stockholm International Peace Research Institute (SIPRI). (2021). Crecen los arsenales nucleares mundiales mientras los Estados prosiguen con su modernización - lanzamiento del nuevo anuario del SIPRI. https://sipri.org/sites/default/files/2021-06/wnf_pr_2021_esp.pdf

Ticehurst, R. (1997). La cláusula de Martens y el derecho de los conflictos armados. Revista internacional de la Cruz Roja, 22(140), 131141. https://www.icrc.org/es/doc/resources/documents/misc/5tdlcy.htm US Department of the Army. (2019). FM 6-27 - MCTP 11-10C. The Commander's Handbook on the Law of Land Warfare. https://armypubs. army.mil/epubs/DR_pubs/DR_a/pdf/web/ARN19354_FM\%20 6-27\%20_C1_FINAL_WEB_v2.pdf (80) 